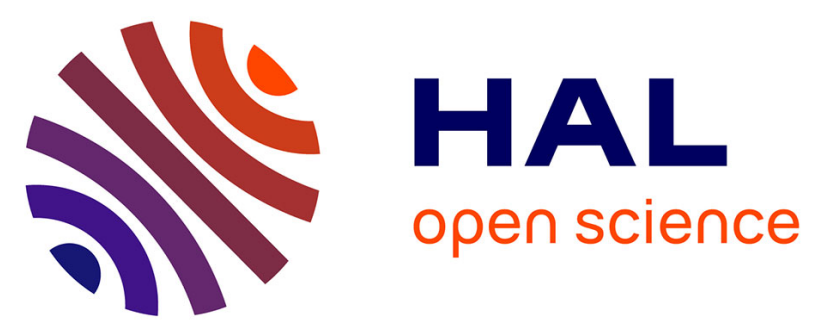

\title{
Le bon, la brute et le migrant? Le rôle négocié des acteurs religieux dans un dispositif policier d'enfermement des étrangers en Allemagne
}

Mathilde Darley

\section{- To cite this version:}

Mathilde Darley. Le bon, la brute et le migrant? Le rôle négocié des acteurs religieux dans un dispositif policier d'enfermement des étrangers en Allemagne. Sociologie du Travail, 2014, 56 (4), pp.472-492. 10.1016/j.soctra.2014.07.008 . halshs-02393798

\section{HAL Id: halshs-02393798 \\ https://shs.hal.science/halshs-02393798}

Submitted on 6 Dec 2019

HAL is a multi-disciplinary open access archive for the deposit and dissemination of scientific research documents, whether they are published or not. The documents may come from teaching and research institutions in France or abroad, or from public or private research centers.
L'archive ouverte pluridisciplinaire HAL, est destinée au dépôt et à la diffusion de documents scientifiques de niveau recherche, publiés ou non, émanant des établissements d'enseignement et de recherche français ou étrangers, des laboratoires publics ou privés. 


\title{
Le bon, la brute et le migrant? Le rôle négocié des acteurs religieux dans un dispositif policier d'enfermement des étrangers en Allemagne
}

\author{
The Good, the Bad and the Ugly Migrant? The Negotiated Role of \\ Religious Actors in a Police Detention and Deportation Centre in \\ Germany
}

\author{
Mathilde Darley* \\ Centre Marc Bloch-CNRS, Friedrichstrasse 191, 10117 Berlin, Allemagne \\ Disponible sur Internet le 16 septembre 2014
}

\begin{abstract}
Résumé
Contrairement au modèle français, le centre allemand de rétention d'étrangers en situation irrégulière étudié ici ne prévoit pas d'autre intervention extérieure que celle des aumôniers. Leur champ d'action déborde cependant largement le cadre de l'assistance spirituelle pour inclure la défense des droits des étrangers retenus et la contestation politique du dispositif d'enfermement. Le huis clos de la rétention met ainsi en scène deux groupes professionnels, aumôniers et policiers, aux objectifs a priori inconciliables. Tout en éclairant les origines de cette cohabitation singulière, $\mathrm{j}$ ' analyserai ici ce que l'intervention d'acteurs religieux dans un dispositif étatique de contrainte fait à la construction, par chaque famille d'acteurs, de sa légitimité professionnelle, et aux pratiques d'assistance et de contrôle qui en découlent.
\end{abstract}

(C) 2014 Elsevier Masson SAS. Tous droits réservés.

Mots clés : Enfermement ; Étrangers ; Rétention ; Police ; Religieux ; Contrôle ; Assistance ; Humanitaire

\begin{abstract}
Contrary to the French model, the German detention and deportation centre for irregular migrants that has been studied here does not allow for any third parties other than chaplains. However, their scope of action goes far beyond spiritual assistance and extends to the defence of detained aliens' rights and political contestation of the confinement regime. The detention centre therefore brings together two professional
\end{abstract}

* 224, rue du Faubourg-St-Martin, 75010 Paris, France.

Adresse e-mail : mathilde.darley@gmail.com 
groups, chaplains and police officers, whose goals are a priori irreconcilable. After explaining the origins of this unique juxtaposition, I will analyse here the impact of the presence of religious actors within the state confinement regime on the construction, by each group of actors, of its professional legitimacy and on the practices of assistance and control that go along with it.

(C) 2014 Elsevier Masson SAS. All rights reserved.

Keywords: Confinement; Migrants; Detention; Police; Chaplains; Control; Assistance; Humanitarian

En Allemagne comme dans la plupart des pays de l'Union européenne, l'arrestation d'un étranger en situation irrégulière est généralement suivie de son placement en rétention administrative, qui doit permettre de préparer et de réaliser son éloignement du territoire. Les conditions de réalisation de la rétention administrative, et notamment les acteurs qui y sont associés, diffèrent cependant largement d'un pays à l'autre.

Dans le dispositif policier de rétention de Großstadt, grande ville allemande où s'est déroulée l'enquête à l'origine de cet article, la société civile est représentée non par des acteurs associatifs (comme c'est le cas en France, voir Fischer, 2009), mais par des membres du clergé ${ }^{1}$. Les aumôniers sont en effet les seuls acteurs non policiers autorisés à pénétrer quotidiennement dans le centre de rétention et, plus encore, dans l'espace des cellules des retenus ${ }^{2}$. Ce «privilège», selon leurs propres termes, trouve sa source dans la Loi Fondamentale allemande (Grundgesetz), dont l'article 7 entérine les relations particulières qu'entretiennent État et religion en Allemagne ${ }^{3}$. Puisant également dans l'héritage est-allemand d'engagement des acteurs religieux aux côtés des détenus et contre l'État, à Großstadt l'intervention des aumôniers, dont la mission est officiellement désignée par le terme de «soin des âmes » (Seelsorge), inclut en pratique un important volet d'assistance juridique visant à contester le placement en rétention des étrangers et leur éloignement du territoire. De son côté, le corps policier comprend les agents en charge de la surveillance des étrangers retenus, mais aussi des travailleurs sociaux et un service médical, dont la mission est néanmoins articulée autour d'un même objectif de maintien du dispositif d'enfermement.

Au regard de cette cohabitation singulière dans le paysage européen de l'enfermement des étrangers, cet article vise à restituer le système d'interactions professionnelles observé à Großstadt

\footnotetext{
${ }^{1}$ Le contexte fédéral allemand conduit à une grande disparité des dispositifs de rétention d'un État fédéré (Land) à l'autre: à Großstadt, le confinement des étrangers en situation irrégulière s'effectue dans un ancien bâti carcéral spécialement affecté à la rétention administrative et contrastant par conséquent avec les dispositifs rétentionnaires d'autres Länder, souvent fondus dans l'institution pénitentiaire. De ce fait, la gestion et le contrôle des étrangers enfermés sont à Großstadt du ressort de la police, et donc du ministère régional de l'Intérieur, et non de celui de la Justice.

${ }^{2}$ Le contexte rétentionnaire de Großstadt se distingue, à la différence de la situation française par exemple, par l'absence de conseillers juridiques ayant systématiquement accès aux dossiers des retenus et assurant la défense de leurs droits. Certes, une permanence gratuite est proposée une fois par semaine par des avocats bénévoles mais, d'une part, le conseil prodigué est «à usage unique » (il vise à rendre un avis sur la nécessité ou non pour le retenu de se faire ultérieurement assister, contre rétribution, par un avocat) et, d'autre part, les avocats impliqués concèdent ne pas maîtriser les logiques selon lesquelles certains retenus leur sont adressés et d'autres pas, comme me l'a expliqué en entretien un avocat spécialisé en droit des étrangers (les modalités de l'enquête et les personnes interviewées font l'objet d'un encadré méthodologique infra).

${ }^{3}$ L'article 7 prévoit notamment la tenue de cours de religion à l'école, le prélèvement d'un impôt destiné aux églises catholique et protestante (Kirchensteuer), ainsi que l'intervention d'aumôniers dans les institutions publiques. L'action religieuse en rétention s'inscrit par ailleurs dans la longue tradition de délégation, par l'État (ouest-)allemand, de certaines de ses missions, sociales notamment, aux églises catholique et protestante et à leurs organisations caritatives (Becci, 2008).
} 
entre ces deux groupes, policiers et aumôniers, aux objectifs a priori inconciliables. À partir d'observations ethnographiques menées aux côtés des aumôniers de Großstadt et d'entretiens conduits avec les différents acteurs du dispositif d'enfermement (voir encadré), et en m'appuyant notamment sur l'appareil théorique de la sociologie des professions fourni par Abbott (1988), Hughes (1996) et Strauss (1992), je chercherai à analyser la manière dont se construisent et se négocient, au quotidien, les légitimités respectives des interventions policière et religieuse dans le centre fermé. Pour ce faire, je reviendrai d'abord sur les conditions dans lesquelles les aumôniers sont progressivement parvenus à négocier une position dans l'ordre des professions en rétention. La professionnalisation de leur fonction qui en a découlé semble cependant également s'être accompagnée d'un brouillage des frontières entre groupes professionnels au sein du dispositif d'enfermement: l'investissement concurrent, par les acteurs religieux et policiers, du registre «humanitaire », témoigne ainsi d'un rapprochement des répertoires mobilisés par les deux groupes d'acteurs ${ }^{4}$, tandis que l'ethos professionnel religieux, articulé autour des valeurs d' « empathie » et de «dignité humaine», semble présenter certaines formes de perméabilité au contrôle exercé au sein du lieu d'enfermement. Cet article interroge donc les effets de cette «expertification» (Lochard et Simonet, 2010) de la figure religieuse en rétention sur l'économie des rôles et des pratiques professionnelles dans un dispositif étatique de contrainte.

\section{L'enquête}

Une enquête ethnographique a été réalisée, neuf mois durant en 2010 et 2011, aux côtés d'aumôniers protestants intervenant à Großstadt auprès des hommes et des femmes retenus. Se déclarant «intéressés par le dialogue avec la recherche », ceux-ci m'ont proposé le statut de stagiaire, couramment endossé dans l'enceinte du centre par les étudiants en théologie se destinant à une carrière d'aumônier. J'ai donc pu suivre, deux à trois fois par semaine, les deux pasteurs, homme et femme, dans leurs visites aux retenus, puis effectuer seule certaines visites. Ce statut de stagiaire constituait un poste d'observation privilégié des interactions non seulement entre aumôniers et retenus, mais aussi entre aumôniers de différentes confessions (notamment catholiques et protestants) ainsi qu'entre aumôniers et acteurs policiers (surveillants, travailleurs sociaux, médecins, psychologue).

À partir de 2012, cette enquête ethnographique a été complétée par des entretiens (seize au total) conduits avec des acteurs intervenant dans les murs du centre de rétention ${ }^{5}$ : neuf policiers dont six surveillants et trois occupant des fonctions plus élevées dans la hiérarchie policière du centre; quatre aumôniers, dont deux protestants et deux catholiques; le chef du service médical policier; la psychologue, dirigeant le département de travail social

\footnotetext{
4 Voir l'utilisation de la notion de répertoire par Stéphanie Vermeersch à propos de l'engagement associatif bénévole «pour désigner l'existence de valeurs accessibles [aux bénévoles] dans le cadre de la légitimation éthique de leur engagement [associatif] » (Vermeersch, 2004, p. 693).

5 Ces entretiens ont été conduits en langue allemande et les extraits présentés ici ont été traduits par l'auteur.
} 
à Großstadt; un travailleur social. Hors les murs, des entretiens ont également été conduits avec un responsable de la politique migratoire régionale, un responsable du département «expulsions» de l'Office des étrangers, un avocat spécialisé en droit des étrangers et un ancien aumônier membre de la «commission statuant sur les cas de flagrante injustice» (voir infra, section 2.2).

\section{Les aumôniers dans le dispositif de privation de liberté : de l'indétermination d'une fonction à la négociation d'une position}

«Les aumôniers n'ont en soi pas de profil de poste. [...] Ils remplissent leur mission comme ils l'entendent, en fonction de ce qu' ils jugent approprié. [...] La conception de leur mission dépend en fait beaucoup de la personnalité de chacun » (Directeur du service médical policier de Großstadt, entretien du 31/10/2012).

Outre l'absence de conventions formellement établies réglementant le cadre d'action des aumôniers en rétention, la marge de manœuvre dont ces derniers disposent dans leurs pratiques quotidiennes d'intervention se traduit notamment par les «privilèges » dont ils bénéficient, selon leurs propres termes, en matière de circulation notamment, dans un dispositif policier dont la surveillance des déplacements constitue pourtant la pierre angulaire. À leur arrivée, les aumôniers ne sont ainsi pas tenus de passer par le portique de détection à métaux et se voient remettre par le poste de garde une clef leur permettant de circuler librement d'un étage à l'autre de la rétention - à la différence des familles des retenus ou même des avocats, dont l'espace d'intervention se cantonne au parloir. Les représentants du clergé passent donc la majeure partie de leur temps dans les cellules auprès des retenus, espace dans lequel les policiers interviennent quant à eux assez peu, sauf en cas de trouble à l'ordre interne du centre. Les aumôniers disposent en outre d'un bureau, qu'ils considèrent, du fait de sa relégation au sous-sol du centre, comme un espace sûr, «à l'abri des oreilles policières ». Enfin, ils ont obtenu l'installation d'une «salle de prières », située à l'étage des cellules d'isolement et dans laquelle a lieu l'office œcuménique hebdomadaire réunissant aumôniers et retenus sans présence policière. Autrement dit, les espaces professionnels dans lesquels évoluent les aumôniers sont pour la plupart peu ou pas investis par les policiers, par ailleurs tenus à l'écart des échanges entre retenus et aumôniers par le principe de confidentialité s'appliquant a priori aux relations entre un croyant et un membre du clergé.

Le centre de rétention de Großstadt met ainsi en scène deux groupes professionnels — policiers et religieux - aux intérêts antagonistes et aux territoires professionnels (Abbott, 1988) a priori distincts, mais que leur coexistence quotidienne dans le huis clos de la rétention conduit ou contraint à composer l'un avec l'autre. Les développements qui suivent viseront donc à éclairer les origines et modalités de cette cohabitation singulière, avant d'interroger leurs effets sur le quotidien de la rétention.

\subsection{Du militantisme à l'expertise : autonomisation de l'intervention religieuse}

«L'année dernière, l'Église a réussi à faire libérer 80 personnes sur environ 800 retenus ! Et $20 \%$ des retenus ont été libérés suite à l'intervention d'un avocat qu'on avait contacté. Donc $30 \%$ des retenus sont sortis grâce à nous. Ça veut dire à la fois que notre travail en rétention a du sens et que $30 \%$ des retenus sont en rétention pour rien » (Markus, aumônier 
catholique, lors d'un échange que nous avons eu durant mes observations sur le terrain, le 16/04/2010).

L'engagement des acteurs religieux de Großstadt en faveur de la libération des retenus et, partant, contre le dispositif étatique de privation de liberté, doit avant tout être replacé dans l'histoire des relations mouvementées entre églises et État en RDA, sur l'ancien territoire de laquelle est situé le centre de Großstadt. Bien que soumises au contrôle du parti, les églises (et en particulier l'Église protestante) constituaient en RDA « les seules organisations jouissant d'un degré relatif d'autonomie» et, partant «le principal foyer de la critique» adressée au système politique (Thériault, 2006). Dans ce contexte, le positionnement des acteurs religieux est généralement apparu indissociable de formes d'engagement fortement politisées, parmi lesquelles la défense des droits des détenus occupait une place de premier ordre (Becci et Willems, 2009). À l'Ouest, l'Église catholique s'est engagée dès la fin de la Seconde Guerre mondiale dans l'aide aux réfugiés ${ }^{6}$, dont elle a fait un de ses principaux domaines d'intervention dans la vie politique et sociale en RFA. Si la réunification a fait des églises catholique et protestante des partenaires institutionnels du nouvel État allemand - lequel contribue d'ailleurs en grande partie à leur financement - l'intervention des aumôniers dans les lieux de privation de liberté s'inscrit néanmoins aujourd'hui dans un contexte de prises de position régulières des églises sur des questions publiques, et donc de rapport de tension variable avec l'État (Thériault, 2006). Comme les aumôniers étudiés dans d'autres contextes de privation de liberté, et notamment dans les prisons de l'ex-Allemagne de l'Est (Becci et Willems, 2009), les acteurs religieux de Großstadt, âgés de cinquante à soixante ans et ayant fait l'expérience de l'Allemagne divisée, affirment et affichent donc de manière récurrente leur sympathie pour les retenus et leur distance vis-à-vis des membres du personnel policier de surveillance. Ces derniers se caractérisent par ailleurs souvent par un rapport critique à la religion : pour la plupart originaires d'Allemagne de l'Est et ayant grandi sous le régime socialiste est-allemand, un des plus sécularisés d'Europe (Thériault, 2006), les policiers accueillent généralement la pratique religieuse des aumôniers avec une ironie potentiellement héritée de leur socialisation socialiste (Becci et Willems, 2009).

Journal de terrain, 02/05/2011 — En entrant dans la salle de prières pour reconduire dans leurs cellules les retenus ayant participé à la messe hebdomadaire, un policier s'exclame : «Mais ça pue là-dedans, qu'est-ce que vous avez fumé ?». Elisabeth, pasteure, lui répond sèchement et visiblement offusquée qu'on y a brûlé de l'encens. Le policier lance alors : «Ah, dans ces moments-là, je comprends vraiment pourquoi je ne veux rien avoir à faire avec l'Église », et tourne les talons.

Outre l'épaisseur historique du rapport entre institutions étatiques et acteurs religieux, les trajectoires sociale et professionnelle des aumôniers catholiques et protestants de Großstadt éclairent également leur positionnement en rétention, et notamment l'extension de leur mandat au-delà du champ religieux et leur implication aux côtés des retenus. Les acteurs dont il est ici question se sont en effet pour la plupart engagés très tôt, dans le cadre de leur professionnalisation religieuse, aux côtés des étrangers, et en particulier de ceux perçus comme les plus vulnérables, tels les demandeurs d'asile et les sans-papiers : il s'agissait pour eux d'être «la voix de ceux qui n'en ont pas », au nom de la défense de la «dignité humaine ». Ainsi, Peter, âgé d'une cinquantaine d'années et intervenant en tant qu'aumônier protestant dans le centre de rétention de Großstadt

\footnotetext{
${ }^{6}$ Sous la forme, dans un premier temps, d'un concours de l'Église à l'assistance procurée aux Allemands expulsés d'Europe de l'Est; à ce sujet, voir Voßkamp, 2007.
} 
depuis sept ans, était très impliqué dans les mouvements de jeunesse protestants pour la paix. Il obtient sa première paroisse au début des années 1990, juste après la réunification, dans une ville d'ex-Allemagne de l'Est. Il s'engage alors dans la lutte contre le racisme et travaille auprès de demandeurs d'asile et de réfugiés. Au milieu des années 2000, il pose sa candidature au poste d'aumônier dans le centre de rétention de Großstadt, où sont alors détenus près de 300 étrangers en attente d'expulsion. Au sein du dispositif de rétention, Peter assigne aux offices religieux « une dimension religieuse, mais aussi politique ${ }^{7}$, conférant ainsi un sens politique à son action alors même que celle-ci s'ancre hors du champ politique ${ }^{8}$. Peter est d'ailleurs également engagé au sein d'une organisation non gouvernementale militant contre la rétention et se distingue par ses fréquentes prises de position critiques, y compris dans les médias, à l'égard de la politique de contrôle et d'enfermement des étrangers. À ce titre il place, à l'instar des autres religieux de Großstadt, le conseil juridique aux retenus au cœur de son travail d'aumônier, qu'il définit comme celui d'un «gardien des droits de l'homme» en rétention.

Elisabeth quant à elle, soixante ans environ, rend visite une ou deux fois par semaine, depuis onze ans, aux femmes retenues. Elisabeth dit être devenue pasteure «par erreur» ${ }^{9}$. Le bac en poche, elle ambitionne d'abord d'effectuer des études de médecine mais constate qu'en RDA, d'où elle est originaire, ce projet de carrière lui est inaccessible. C'est au cours de conversations avec ses amis, pour la plupart originaires des cercles de jeunesse protestants au sein desquels elle est très engagée, qu'elle décide de s'inscrire en théologie à l'université, avant d'émigrer en Allemagne de l'Ouest au cours de ses études. Ses premières tentatives pour s'insérer dans le pastorat, qui ne s'est ouvert aux femmes que quelques années plus tôt, sont cependant marquées par un sentiment de profonde injustice lié au traitement différencié dont femmes et hommes pasteurs font l'objet. Suite à de nombreuses candidatures, Elisabeth obtient finalement un poste au sein de la paroisse d'un hôpital, limité à six ans et supprimé à l'issue de cette période. Sans ministère, Elisabeth bénéficie d'un statut spécial lui permettant de continuer à percevoir son traitement mais qui la contraint à accepter différents remplacements en fonction des nécessités. C'est dans ce contexte qu'elle est envoyée par l'Église protestante dans le centre de rétention de Großstadt. Cette affectation d'office ne lui plaît pas, d'autant qu'elle a «toujours dit qu' [elle] ne voulai[t] pas aller en prison ». Elle dit avoir «peur de la police», dont elle décrit les comportements comme guidés uniquement par «le respect de la consigne». À plusieurs occasions, elle ne manque pas de signaler, par des actes symboliques de résistance (refus de laisser contrôler son sac à l'entrée par exemple), sa volonté de ne pas se soumettre au pouvoir policier des lieux. Elle rechigne par ailleurs à célébrer l'office religieux en rétention, au motif qu'elle a «du mal à dire aux autres comment ça marche dans le ciel et sur la terre».

Autrement dit, le dépassement de la seule vocation religieuse de leur action constitue un socle commun à l'ensemble des aumôniers catholiques et protestants intervenant à Großstadt, que cette forme de transcendance résulte de formes très précoces de politisation de leur rôle (comme dans le cas de Peter) ou d'une volonté de mise à distance du religieux (comme chez Elisabeth).

«En tant qu'aumônières et aumôniers, nous travaillons en faveur des personnes qui s'adressent à nous. Cela ne signifie pas qu'il n'existe pas de règles objectives et scientifiques pour le travail d'aumônier. Nous aidons aussi bien les personnes qui souhaitent

\footnotetext{
${ }^{7}$ Les éléments de trajectoire repris ici sont issus de l'entretien conduit avec Peter le 27/02/2012.

8 Processus que Jacques Lagroye (2003) analyse comme un « dépassement des limites ».

${ }^{9}$ Les éléments de trajectoire repris ici sont issus de l'entretien conduit avec Elisabeth le 10/05/2012. Un portrait plus complet de cette aumônière a été dressé dans Darley, 2012.
} 
rentrer rapidement dans leur pays que celles qui nous demandent de nous assurer que leur placement en rétention et leur expulsion sont juridiquement corrects. Nous travaillons étroitement, pour évaluer la situation juridique, avec différents avocats. C'est l'homme dans son entier — ses dispositions religieuses, sa forme physique et ses états d'âme — qui est pour nous au centre de l'attention. Notre mission est de l'aider à retrouver une forme de sécurité, à arrêter les bonnes décisions, à prendre conscience de sa propre existence » (Lettre adressée par les aumôniers au service médical policier, avril 2011).

L'inscription par les aumôniers de leur intervention dans un répertoire d'actions dépassant la seule assistance spirituelle, pour inclure notamment le conseil juridique, leur permet alors à la fois de se présenter comme « utiles » et de produire « des discours opérant une montée en généralité »et, ce faisant, contribue à la « politisation » de leur rôle (Hamidi, 2006). Les offices œcuméniques hebdomadaires célébrés alternativement par le pasteur protestant ou le prêtre catholique en présence de tous les aumôniers catholiques et protestants constituent à cet égard des espaces particuliers en rétention: explicitement présentés comme des espaces d'articulation des niveaux d'engagement « religieux » et «politique », ils permettent aux aumôniers, en l'absence de contrôle policier, de s'ériger «contre le système» d'enfermement des étrangers. Ce discours supra-confessionnel et politique a d'autant plus de chances de s'implanter dans le contexte des offices que la plupart des retenus (généralement nombreux) y assistant ne sont de confession ni catholique ni protestante ${ }^{10}$ : davantage qu'un lieu de culte, la salle de prière est avant tout pour eux un espace permettant de sortir du confinement en cellules et de «structurer le temps carcéral» (Sarg et Lamine, 2011), tout en ayant accès à une forme de socialisation genrée mixte par ailleurs prohibée dans le dispositif rétentionnaire ${ }^{11}$. La dimension transconfessionnelle de l'intervention religieuse en rétention, sans être spécifique au contexte rétentionnaire de Großstadt ${ }^{12}$, se traduit également par le fait que l'évaluation de l'engagement politique et juridique en faveur des retenus constitue une ligne de fracture bien plus prégnante, entre aumôniers, que l'appartenance confessionnelle. Ainsi, tandis que le refus d'Elisabeth de célébrer les offices n'est pas contesté par ses collègues protestants ou catholiques, son investissement jugé insuffisant auprès des retenus et son goût pour les vacances fréquentes et dans des conditions jugées «luxueuses » par les autres aumôniers motivent des critiques récurrentes à son encontre, questionnant notamment sa capacité à s'engager aux côtés de populations « opprimées».

\footnotetext{
${ }^{10}$ Dans le centre de rétention de Großstadt, les Vietnamiens, les ressortissants d'ex-URSS et d'ex-Yougoslavie, les Turcs et, dans une moindre mesure, les ressortissants de pays nord-africains, figurent parmi les nationalités les plus représentées. Les observations des offices religieux célébrés en rétention laissent penser que la part des retenus protestants ou catholiques est nettement inférieure à celle des retenus d'autres confessions (orthodoxes, musulmans ou bouddhistes notamment). Cependant, à Großstadt comme dans d'autres lieux d'enfermement, les retenus semblent faire preuve d'une activité religieuse (si l'on entend par là le fait de participer aux offices) remarquablement élevée pouvant s'expliquer non seulement par le caractère quasi incontournable des aumôniers entre les murs, mais aussi par leur participation active à l'amélioration des conditions pratiques et psychologiques de la privation de liberté — par l'achat de cartes SIM pour les téléphones portables, la prise de contacts avec la famille ou les amis des retenus, ou simplement leur disponibilité pour toute forme d'échange ou de confidence. Sur le rôle des aumôniers en prison, voir aussi Beckford, 2001.

11 Sur les offices comme espaces d'exception dans l'ordre genré de la rétention, puisqu'ils constituent les seuls lieux autorisant la réunion de retenus des deux sexes, voir Darley, 2012.

12 Ainsi, la conférence « Conseil et aumônerie en rétention » organisée à Vienne en janvier 2011 réunissait des aumôniers catholiques et protestants originaires d'Allemagne, de Suisse et d'Autriche et visait précisément à interroger les conditions d'exercice d'une activité de conseil (notamment juridique) aux étrangers par les aumôniers intervenant en rétention dans ces différents pays.
} 
Le «travail incessant de rationalisation des pratiques » auquel se livrent les aumôniers intervenant en rétention, visant à établir «une relation aussi étroite que possible entre leurs actes et les savoirs transmis par l'institution » pour légitimer leur intervention dans un dispositif étatique de privation de liberté, mobilise ainsi une référence au «message évangélique et à l'éthique chrétienne, autant [qu'à] l'attention privilégiée que l'Église doit accorder aux pauvres, aux humiliés, aux victimes de la société et aux étrangers » (Lagroye, 2006). En témoignent notamment les sermons prononcés lors des offices :

«Personne ne doit être exclu — et vous ne devez pas non plus être exclus de la vie en société. Dieu est notre arbitre. Il nous montre le carton rouge ou jaune quand il y a faute - lorsque nous blessons les autres ou que nous ne respectons pas les règles du fair-play. L'expulsion, ce n'est pas fair-play, et les hautes clôtures de sécurité autour de vous ne le sont pas non plus. Je souhaite que les jeux soient justes. [...] Que personne ne soit exclu du jeu. Dieu ne vous exclut pas. Il veut que vous ayez votre chance dans la vie. Dieu vous bénisse. Amen » (Sermon prononcé par Peter, aumônier protestant, à l'occasion du lancement de la coupe du monde de football, 11/06/2010).

En invoquant à la fois le principe suprême de la Loi Fondamentale allemande (la défense de la dignité humaine, énoncée à l'article $1^{\mathrm{er}}$ de la Grundgesetz) et une légitimité divine comme ressorts de leur action, les aumôniers adjoignent donc la gestion des biens légaux à la définition étroite de leur rôle comme gestion des biens de salut (Bourdieu, 1971). Ce faisant, ils justifient leur position a priori «oblique» par rapport à l'agencement juridique du monde des hommes et contribuent à asseoir leur autonomie dans un contexte professionnel $a$ priori hostile à l'intervention religieuse.

\subsection{Entre contraintes, contournements et négociations : faire sa place au sein d'un dispositif en tension}

Journal de terrain, 16/04/2010 — Peter, pasteur, est convoqué dans le bureau du directeur policier du centre. Il en revient plus d'une heure plus tard, assez fébrile, et expose dans le bureau des aumôniers les deux motifs qui ont justifié sa convocation : d'abord, les policiers ont trouvé sur un retenu deux photos de lui-même et de ses co-retenus, prises dans sa cellule alors que les appareils photos et les téléphones portables munis de caméras sont interdits en rétention. Une brève enquête interne leur a permis d'établir que les photos avaient été prises par l'ancien stagiaire du pasteur. Ensuite, Peter a initié et organisé une visite du centre de rétention par l'expert de la sécurité intérieure d'un parti politique représenté au Parlement et a proposé à ce dernier, à l'issue de sa visite, une rencontre avec les aumôniers dans la salle de prières. Le directeur du centre de rétention a donc convoqué Peter pour lui «rappeler qu'il ne pouvait disposer librement des locaux [et] que le pouvoir décisionnel revenait exclusivement » au directeur policier, en concluant par : «Moi qui ne vous ai jamais rien refusé, pourquoi me faites-vous cela?».

Autant qu'il témoigne des marges de manœuvre, de négociation ou de subversion de la règle par les aumôniers au sein du dispositif d'enfermement, ce récit ethnographique rend particulièrement visibles les rappels à l'ordre par lesquels le pouvoir policier réaffirme son autorité sur les lieux. Dans cet univers professionnalisé concurrentiel ${ }^{13}$, c'est précisément la liberté consentie

\footnotetext{
13 Anselm L. Strauss définit un «lieu professionnalisé» comme «un site géographique où des personnes issues de différents horizons professionnels se trouvent rassemblées afin d'y mener à bien leurs objectifs respectifs » (Strauss, 1992, p. 90).
} 
aux aumôniers qui est souvent mobilisée par la direction policière pour qualifier d'abusifs certains comportements des religieux, notamment lorsqu'ils portent en eux la possibilité d'une remise en cause de l'ordre hiérarchique établi et de la clôture du lieu vis-à-vis de l'extérieur. Le crédit que leur statut d'acteurs religieux dans un univers policier coercitif leur confère a priori dans la sphère associative, politique et médiatique ${ }^{14}$ leur permet en effet de faire «sortir des murs » certaines informations présentant un potentiel déstabilisant pour l'autorité policière, par le biais de «méthodes classiques de l'agitation publique» (Sawicki, 2003) telles que sollicitations de la presse, conférences, réunions d'information, etc. Or la mise au jour des « savoirs coupables » ${ }^{15} \mathrm{de}$ l'institution étatique (grève de la faim ou tentative de suicide des retenus par exemple) contribue potentiellement à transposer le régime d'énonciation dans lequel le dispositif d'enfermement des étrangers est habituellement thématisé, le passage d'un registre sécuritaire à un registre compassionnel risquant alors de mettre à mal la légitimité du contrôle étatique (Siméant, 1993, 1998 ; Fischer, 2005).

Les « capacités subversives inédites » (Israël, 2003) dont disposent les aumôniers du fait de leur positionnement critique - en tant que représentants de la société civile dans les murs et « caution religieuse » dans un dispositif policier — justifient dès lors le soupçon permanent dont ils font l'objet dans le discours policier, et les limitations ponctuelles apportées à leur autonomie. Ainsi, l'implication supposée des aumôniers dans certaines tentatives d'évasion motive l'obligation qui leur est désormais faite, lorsqu'ils pénètrent dans l'espace des cellules des retenus, de remettre aux policiers de l'étage la clef qui leur permet de circuler librement dans le centre. Cette nouvelle règle les place de fait dans une position de subordination à l'égard des policiers de garde.

Journal de terrain, 22/12/2010 - Après avoir rendu visite aux retenus, Peter et moi nous postons à la grille et, à l'instar des retenus qui ont besoin d'un renseignement ou souhaitent prendre une douche, tentons d'attirer l'attention des policiers. Confinés dans leur bureau situé dans un renfoncement du palier, ils ne peuvent nous voir, mais devraient nous entendre par la porte restée ouverte. Nous répétons nos appels à leur adresse, d'une voix de plus en plus forte : «Sortie s'il vous plaît !» Au bout d'un temps qui nous paraît long, un policier arrive et nous lance, non sans provocation : «Ben alors, quoi, vous voulez déjà sortir? Vous ne restez pas finalement?».

Face à ces mises en scène policières de l'ordre hiérarchique des lieux, de nombreuses interactions témoignent de formes d'intériorisation, apparente au moins, des normes de l'institution par les aumôniers. Le respect formel des règles — refermer chaque porte à clef, y compris dans les espaces les plus reculés du centre par exemple — voire la déférence témoignée à l'égard des policiers — tels les remerciements après un contrôle inopiné de leurs sacs ${ }^{16}$ — semblent toutefois s'inscrire ici dans une stratégie plus globale de «conformisme institutionnel» (Dubois, 2003) visant, pour les aumôniers, à relativiser les effets de domination résultant du contrôle policier des

\footnotetext{
${ }^{14}$ Les articles régulièrement consacrés, dans la presse, au centre de rétention de Großstadt illustrent ainsi le crédit $a$ priori accordé à l'intervention religieuse, présentée comme «la seule bribe de normalité » (Süddeutsche Zeitung, 01/07/2010) dans le dispositif policier d'enfermement des étrangers.

15 Everett C. Hughes dit des savoirs coupables : «Leur caractère coupable peut tenir à ce que le profane serait obligé de les divulguer, ou à ce que leur dissimulation entacherait aux yeux des autorités ou du public, ou pourrait entacher, l'intégrité morale de celui qui les détient» (Hughes, 1996, p. 101).

16 Journal de terrain, 09/06/2010 - À l'issue d'un contrôle des sacs ordonné par le policier de garde, Peter lance au policier qui lui rend son sac: «super super, merveilleux, merci merci !» avant d'ajouter à voix basse, à mon intention : «ils en font un cirque aujourd'hui ! ».
} 
lieux par l'obtention de marges de manœuvre, voire même la transgression d'interdits édictés par le règlement.

Journal de terrain, 16/04/2010 - Un retenu kosovar vient d'être réadmis dans le centre, de mauvaises conditions météorologiques ayant empêché son expulsion. Lorsque Peter et moi lui rendons visite dans sa cellule, il nous raconte que, lors de la fouille à corps accompagnant sa réadmission dans le centre, les policiers lui ont confisqué 300 euros trouvés dans ses chaussures, dont 100 euros procurés par Peter auprès de la famille du retenu par le biais d'un transfert international d'argent. Peter m'explique qu'il n'a en principe pas le droit d'apporter de l'argent aux retenus mais qu'il le fait quand on le lui demande, pour éviter que les gens dont l'expulsion ou la libération est imminente se retrouvent dehors sans argent. Il demande aussitôt à deux autres retenus de faire disparaître définitivement le reçu qu'il leur a apporté à l'issue du transfert d'argent parce que son nom y figure et qu'il «risque d'avoir de très gros ennuis » si les policiers le trouvent. Ils réfléchissent ensuite ensemble au meilleur endroit où cacher l'argent en cas de fouille à corps par la police.

Les libertés d'action et de circulation dont jouissent les aumôniers sont donc le fruit de négociations permanentes avec l'autorité, faites à la fois de résistances, de contestations et d'adaptations à l'institution et à ses contraintes. C'est en effet la combinaison de ces différentes manières d' « habiter» le rôle d'aumônier en rétention et de «faire avec» l'institution qui permet aux religieux de préserver voire d'étendre leur autonomie professionnelle. L'étude des formes de frictions, mais aussi de rapprochements, qui en découlent entre policiers et aumôniers invite alors à questionner la possibilité de l'émergence et/ou du maintien d'un «ordre négocié » (Strauss, 1992) dans une institution où se rejoue quotidiennement la tension entre État social et État pénal.

\section{Entre «expérience» policière et «expertise » religieuse : la définition des rôles policier et religieux en rétention}

Si toute profession s'organise autour de la «monopolisation (ou revendication du monopole) d'un domaine d'activité et du savoir qui s'y applique» (Monjardet, 1996, p. 120), la question du monopole de la gestion et de l'organisation du centre de rétention de Großstadt est a priori tranchée par l'État en faveur de la profession policière. Différents travaux ont toutefois mis en évidence, d'une part, l'influence de l'intervention d'acteurs dits «profanes », dans une organisation professionnelle, sur les processus de division du travail et de négociation de l'ordre qui s'y opèrent (Strauss, 1992) et, d'autre part, l'impact, sur un système de professions, des relations de concurrence entre groupes professionnels (Abbott, 1988). Partant de ces acquis de la sociologie des professions, il s'agira d'analyser ici la manière dont chaque groupe professionnel (policiers et aumôniers), en revendiquant le monopole de certaines compétences particulières, cherche à imposer sa légitimité et son savoir-faire face à l'autre.

\subsection{La «concurrence humanitaire»}

Policiers et aumôniers occupent a priori des fonctions bien distinctes dans l'organisation du quotidien de la rétention: il s'agit là de professions établies de longue date, auxquelles sont associées des «carrières professionnelles » et des «idéologies » (Strauss, 1992) particulières qui facilitent l'identification de «territoires de compétences » (Abbott, 1988) a priori bien délimités. Les policiers du centre de rétention sont ainsi affectés soit à la garde du poste de contrôle situé au rez-de-chaussée du bâtiment principal, soit à la surveillance des retenus aux étages. Ils prennent en 
charge les nouveaux retenus conduits au centre, les soumettent à une fouille à corps, confisquent leurs objets de valeur et leur argent, leur attribuent un numéro de retenu puis un lit dans une cellule, selon des «formalités d'admission» caractéristiques du rapport asymétrique entre personnel et reclus dans les institutions dites «totales» (Goffman, 1968). Le rôle des policiers s'inscrit donc dans une définition classique de l'institution policière en tant qu' " institution en charge de détenir et de mettre en œuvre les ressources de force décisives dans le but d'assurer au pouvoir la maîtrise (ou régulation) de l'usage de la force dans les rapports sociaux internes » (Monjardet, 1996, p. 20). De fait, leur intervention se concentre à première vue sur les corps des retenus, «matière humaine » (Benguigui et al., 1994) qu'il ne s'agit cependant pas seulement de discipliner, mais aussi, dans les termes des policiers, de «maintenir en bonne santé ${ }^{17}$. Ce souci minimal des corps constitue en effet le « contrat de base » (Strauss, 1992) de l'institution fermée, dont elle ne saurait faire l'économie sans voir sa légitimité politique remise en cause (Fischer, 2005).

A contrario, la mission des intervenants religieux telle qu'elle est énoncée par l'intitulé de leur poste dépasse le seul souci des corps enfermés pour se concentrer sur les «âmes » des retenus. Dans leur présentation de soi, les aumôniers mettent notamment l'accent sur leurs dispositions «empathiques » à l'encontre des retenus, dispositions dont ils déplorent de manière récurrente l'absence chez les acteurs policiers ${ }^{18}$.

Journal de terrain, 15/04/2011 - Insatisfaits du fonctionnement du service médical policier en rétention, les aumôniers ont ces derniers temps à plusieurs reprises dénoncé, par voie de presse notamment, les mauvaises conditions d'accès aux soins des étrangers retenus à Großstadt et les «dérives » ayant par exemple conduit à l'expulsion d'étrangers malades. La tension entre aumôniers et personnel médical policier est donc forte. Depuis plusieurs semaines, les aumôniers sollicitent auprès du chef policier du service médical et du médecin en poste un entretien visant à «développer une charte pour la coopération entre personnel médical et aumôniers en rétention ». Les premières tentatives ont essuyé un refus de la part des autorités médicales, reprochant aux aumôniers leur approche «subjective» et «émotionnelle» de la rétention et des retenus. Une rencontre a finalement lieu entre les aumôniers, la direction policière du centre, le chef policier du service médical et le médecin. L'ordre du jour a été établi par les aumôniers et aborde l'introduction d'un examen médical et psychologique systématique à l'arrivée des retenus à Großstadt, le droit de regard des aumôniers sur les dossiers médicaux des retenus, la définition médicale des contreindications à la rétention ou à l'expulsion et la clarification du mode d'administration des traitements aux retenus, laquelle donne notamment lieu à l'échange suivant :

- Peter (en préambule) : Nous, on aimerait bien connaître exactement les critères médicaux de jugement. De notre côté, on peut juger d'un point de vue émotionnel ou humain, mais quelle est la vision médicale des choses? Par exemple, pouvez-vous nous préciser quels médicaments sont administrés aux retenus au service médical? J'ai vu récemment que les antibiotiques étaient distribués à l'étage par les policiers alors que je pensais qu'ils ne l'étaient qu'au service médical ${ }^{19}$.

\footnotetext{
17 Entretiens avec un surveillant policier (16/01/2012) et un travailleur social policier (26/01/2012).

18 Sur la «norme de l'empathie », voir aussi le travail de Vincent-Arnaud Chappe (2010) sur les militants d'une association antiraciste.

19 Pour les médicaments administrés «à l'étage», c'est-à-dire dans l'espace des cellules et par les policiers (et non le personnel médical), la procédure est la suivante : les médicaments sont stockés dans une armoire située à côté du poste de contrôle et les policiers disposent d'une liste faisant état de la prescription pour chacun des patients sous traitement. En
} 
- Chef du service médical policier: Oui mais enfin bon, il faut reconnaître une certaine autonomie aux gens ! Ils avaient aussi une vie dehors, avant d'être placés en rétention!

—Médecin: Si je soupçonne qu'un retenu ne prend pas correctement ses médicaments, j'indique qu'ils doivent lui être donnés en bas, au service médical. Et certains médicaments, comme les calmants et les somnifères, ne sont donnés qu'en bas à cause du risque que les retenus les collectent dans leur cellule.

- Peter : Oui oui, je comprends, de toutes façons si ça ne tenait qu'à moi je préférerais que tout soit donné en bas.

- Chef du service médical policier: Mais c'est dégradant pour la dignité des retenus! Dehors ils ont aussi eu une vie, il faut respecter ça! Il n'y avait personne pour leur beurrer leur tartine tous les matins ! [...]

En sortant de l'entretien, les aumôniers organisent un débriefing dans leur bureau. Peter fait remarquer que son but est toujours d'amener le service médical policier à «définir ce que cela signifie de travailler comme médecin dans un lieu comme un centre de rétention » et lance: «c'est quand même incroyable à quel point ils manquent d'empathie !». Au mot «empathie», les autres rient et font remarquer que ce terme ne fait sans doute pas partie du vocabulaire des médecins du centre.

L'interaction décrite ci-dessus éclaire à différents égards l'analyse des mondes professionnels policiers et religieux. Lorsqu'ils mettent en avant le caractère «émotionnel » et «empathique » de leur démarche, les aumôniers cherchent ainsi à atteindre un double objectif : non seulement confirmer l'autorité policière dans son rôle de «professionnel » face aux catégories de jugement «profanes » des religieux — et négocier ainsi, à partir d'une apparente déférence vis-à-vis de l'institution policière, d'éventuelles marges de manœuvre dans le débat -, mais aussi souligner, en creux, qu'il s'agit précisément là de dispositions qui font défaut aux acteurs policiers et asseoir ainsi leur supériorité morale. Or c'est justement sur ces dispositions «émotionnelles » et «empathiques» des aumôniers que les acteurs policiers s'appuient, dans le quotidien de la rétention, pour délégitimer leurs pratiques de jugement et, ce faisant, contester le monopole qu'ils revendiquent à «faire le bien » entre les murs : les acteurs du contrôle font en effet de la propension des aumôniers à l'empathie, empreinte de charité chrétienne, un facteur d'altération de leur discernement. Ils sont ainsi décrits comme «trop bons», «manquant d'objectivité », «instrumentalisés par les retenus [qui] exploitent leur angélisme», quand les policiers mettent au contraire en avant les savoirs qu'ils tirent de leur «expérience».

Si l'extrait de journal de terrain présenté ci-dessus illustre bien les modes de construction concurrentiels d'une légitimité professionnelle de l'action en rétention, il met cependant également en lumière certains parallèles entre les deux corps de métiers. De manière saisissante, alors que les religieux font de l'empowerment des migrants, de l'empathie et de l'émotion des principes fondamentaux de leur action, celle-ci apparaît non exempte de velléités de contrôle, ce que ne manquent pas de souligner les acteurs policiers : le reproche de négation de l'autonomie des retenus, formulé ci-dessus par le chef du service médical policier, revient ainsi à ramener

général, un policier entre dans une cellule avec le nombre exact de cachets à administrer et appelle : «numéro 422 ? ». Le retenu ainsi désigné doit alors ingérer les cachets en présence du policier. Il arrive toutefois régulièrement que l'autorité médicale réalise a posteriori qu'un retenu n'a pas pris le traitement prévu pour lui (ou en a pris un autre). Pour les traitements les plus lourds, ou les retenus dont on craint qu'ils se soustraient au contrôle policier et tentent de stocker les médicaments dans leur cellule, le médecin peut donc prescrire une administration à l'infirmerie : celle-ci nécessite que le retenu soit accompagné par un policier au rez-de-chaussée, où le personnel médical (infirmiers ou médecin) prend en charge l'administration du traitement. 
le rôle des acteurs religieux, et l'autorité qu'ils revendiquent sur les lieux, au projet originel de contrôle et d'amendement des comportements sous-tendant l'intervention religieuse en milieu fermé (Beckford, 2001). Les médecins de l'institution policière recourent à des modes de légitimation de leurs pratiques reposant non seulement sur une forme de discrédit du jugement religieux, mais aussi sur la mobilisation de valeurs jusqu'alors présentées par les aumôniers comme des prérogatives mêmes de l'action religieuse en rétention : ainsi, par un effet de retournement du stigmate, le chef policier du service médical argue de la nécessaire défense de la « dignité humaine » des retenus pour jeter le discrédit sur l'évolution des pratiques réclamée par les aumôniers en matière d'administration des traitements aux retenus. Tant le glissement des termes du discours policier observé ici que les revendications de contrôle qui se dessinent de la part des aumôniers invitent donc à questionner plus avant la possibilité d'un renforcement mutuel des discours et des pratiques policières et religieuses.

\subsection{De la contrainte à la compassion: discordance ou concordance des rôles?}

Les entretiens conduits ${ }^{20}$ montrent en effet que c'est aussi souvent par la mise en avant de leurs compétences humaines que les policiers (re)construisent le bien-fondé de leur travail auprès des étrangers retenus face à la revendication religieuse d'un «monopole humanitaire » en rétention. De manière emblématique, la charte édictée par le directeur policier du centre à l'intention des policiers en poste et des visiteurs extérieurs, dont la devise principale est « Aussi peu de contrôle que possible, autant d'accompagnement que nécessaire!», prévoit ainsi en première ligne la « défense de la dignité humaine » - soit précisément le principe même dont on a vu qu'il était au cœur du message évangélique porté par les aumôniers. Contrant par là même les représentations coercitives qui leur sont associées, les policiers de Großstadt déplacent le curseur de la concurrence entre compétences policières et religieuses vers une forme de souci des retenus, une attention à l'humain revêtant certains attributs d'une politique du care (Molinier et al., 2009).

«Ce qui est important, c'est le rapport aux gens. C'est ça en fait l'important, c'est de se comporter humainement avec eux, de toujours considérer l'autre en tant qu'homme [...] pour désamorcer un peu les choses, simplement donner un peu de paix intérieure aux gens [...], parce que nous sommes leurs principaux interlocuteurs. En fait on peut dire que les travailleurs sociaux qui passent après nous n'ont plus grand-chose à faire. On remplit notre mission : faire régner la paix à l'étage, mais aussi la paix intérieure chez les gens » (Policier en charge de la surveillance des retenus, entretien du 17/08/2012).

Autrement dit, les policiers adjoignent à la définition policière de leur rôle la revendication d'un «gouvernement humanitaire » des retenus (Fassin, 2010). Ce faisant, ils contestent la division morale du travail promue par les aumôniers en investissant précisément le registre empathique et les compétences «humaines » et/ou « sociales» dont les aumôniers ont fait leur signe distinctif dans le dispositif policier. En se présentant comme de «petits travailleurs sociaux qui font baisser un peu la pression », comme l'énonce en entretien un policier chargé de la surveillance des retenus, les policiers proposent ici une définition de leur fonction épousant «les termes idéologiques du travail social »(Weller, 1994). Le fait que cette compétence « sociale » soit pourtant habituellement

\footnotetext{
${ }^{20}$ Il convient bien sûr de garder à l'esprit l'incidence de la présence du chercheur sur la construction de cette professionnalité policière : il s'agit ici, pour le policier en situation d'entretien, de «montrer à l'enquêteur que son travail ne se résume ni aux descriptions formelles du poste [...], ni aux représentations que le grand public se fait » de la police et de ses agents (Weller, 1994, p. 29).
} 
largement discréditée au sein de l'institution policière (Bittner, 2001) rend sa revendication dans le contexte professionnel de la rétention particulièrement saisissante ${ }^{21}$. Elle rapproche les agents policiers chargés du maintien de l'ordre en rétention des surveillants étudiés au sein de l'institution carcérale: différents travaux ont ainsi montré que les modes de légitimation des pratiques de maintien de l'ordre par les surveillants de prison reposaient davantage sur la mise en avant de compétences individuelles et subjectives que sur les règles formelles édictées par la hiérarchie (Malochet, 2004). À propos de ces dernières, les policiers de Großstadt disent par exemple : «Il faut une ligne directrice, c'est sûr, mais moi je trouve ça bien qu'on puisse en dévier un peu sur la gauche ou sur la droite ». Le rôle de l' «expérience» comme référentiel mobilisé pour définir les marges de manœuvre et asseoir la pratique professionnelle semble alors particulièrement important. Les policiers s'appuient en effet sur leur âge ( « entre 45 et 60 ans ») et leur expérience (ils sont pour la plupart affectés à Großstadt depuis l'ouverture du centre au milieu des années 1990) pour mettre en avant leurs qualités face aux policiers plus jeunes : ils seraient ainsi «plus équilibrés » dans leurs rapports avec les retenus, «plus cordiaux » et par conséquent capables de pacifier les situations de tension, quand les plus jeunes risqueraient au contraire de se placer dans une logique de confrontation avec les retenus, sensiblement du même âge qu' eux. Cet ethos professionnel se trouve sans doute également renforcé par le fait que les policiers déclarent pour la plupart être entrés dans la police non par goût pour le maintien de l'ordre mais « par hasard » ou par nécessité économique, après avoir exercé d'autres emplois dans d'autres domaines. Ici comme chez les surveillants de prison étudiés par Antoinette Chauvenet par exemple, «l'apprentissage des relations humaines et de la "bonne distance" » permis par l'expérience est donc considéré comme la «formation de base du métier », permettant d'éviter les troubles et de temporiser (Chauvenet, 1993).

Or, en matière de prévention du désordre, les acteurs du contrôle considèrent souvent les aumôniers non pas (seulement) comme des agitateurs, mais comme des «partenaires ». On leur attribue en effet des vertus pacificatrices en rétention en raison de l'occupation qu'ils procurent aux retenus — gage d'absence de désordre (Chauvenet, 1993) — mais aussi d'une forme de division technique du travail (Hughes, 1996) qui fait d'eux les agents du bien-être, spirituel notamment, des migrants enfermés.

«Les aumôniers sont d'une valeur inestimable. Pour dire clairement les choses, ils nous enlèvent beaucoup de pression ici — euh, parce que les gens, avec les soucis qui leur pèsent sur l'âme, je veux dire au vrai sens du terme, ben là ils ont quelqu'un qui les prend en charge. On a certes les travailleurs sociaux, mais ils seront toujours un peu étiquetés “État" » (Directeur policier de la rétention, entretien du 14/11/2011).

Autrement dit, les acteurs religieux apparaissent ici également comme au service de l'ordre interne du lieu d'enfermement : au sein d'un dispositif où le pouvoir policier repose quasi exclusivement sur sa capacité à se « saisir des corps » à des fins d'éloignement du territoire (Fischer, 2005 ; Darley, 2008), l'apaisement que les aumôniers procurent aux retenus semble en effet également faciliter leur contribution au projet d'expulsion des autorités.

«Ça, la direction du centre nous le dit parfois directement : on contribue bien sûr à maintenir le calme... Si tu es là, que tu parles avec eux [les retenus], que tu fais l'intermédiaire, que

\footnotetext{
${ }_{21}$ Au cours des différentes enquêtes ethnographiques que j'ai pu effectuer au sein de la police en France, en Allemagne ou en Autriche, l'affirmation « on n'est pas des travailleurs sociaux » est également apparue récurrente dans les discours des policiers.
} 
tu désamorces les conflits... Bon, c'est sûr qu'on peut se demander si c'est ça qu'on veut ou s'il ne vaudrait pas mieux... [...] Qu'est-ce que je fais ici ? Est-ce que je ne travaille pas aussi pour l'Office des étrangers, pour qu'ils puissent expulser plus facilement et dans le calme ? Hm... » (Elisabeth, aumônière protestante, entretien du 10/05/2012).

Redoutant certaines formes de «contamination morale» (Goffman, 1968) d'un corps professionnel à l'autre, du «sale boulot» (Hughes, 1996) de contrôle policier vers les missions plus nobles d'assistance des aumôniers, ces derniers veillent alors à se distinguer de l'autorité policière qui régit les lieux. Après avoir substitué leurs propres badges à ceux distribués par la direction du centre, dont la mention «Direction policière de Großstadt, Peter B., aumônier » leur semblait induire une subordination hiérarchique des aumôniers au pouvoir policier, ils prennent également soin de se distancier, lors de leur passage dans les cellules, des acteurs de la chaîne du contrôle.

«Moi, quand j'arrive, je dis “Bonjour, je suis Père C. et je suis envoyé par l'Église catholique. Je ne suis pas de l'Office des étrangers, pas de la police. Est-ce que je peux voir votre dossier s'il vous plaît ?" » (Markus, aumônier catholique, entretien du 30/08/2012).

Cette distanciation apparaît d'autant plus nécessaire que les observations ethnographiques témoignent de certaines formes de brouillage des frontières professionnelles chez les retenus fraîchement arrivés à Großstadt, notamment entre membres de l'équipe policière de travail social et aumôniers. La confusion naît notamment du fait que la nouvelle équipe de travailleurs sociaux a désormais renoncé au port de l'uniforme policier et ne rechigne plus à s'installer brièvement dans les cellules autour d'un thé avec les retenus lorsque le traitement de leur situation nécessite un contact approfondi. Les « anciens » retenus initient cependant rapidement leurs nouveaux coretenus à l'établissement d'une distinction entre police et églises. Au-delà de l'énonciation d'une frontière dans la présentation de soi, les aumôniers se distancient en effet de l'administration policière par l'intérêt qu'ils portent au dossier juridique des retenus, mais aussi par le ton adopté dans leurs échanges avec eux, empreint de sollicitude et de complicité, voire souvent d'humour (voir aussi Bouagga, 2013). Les retenus préfèrent donc généralement faire part de leurs doléances relatives à des besoins matériels et/ou intimes aux aumôniers plutôt qu'aux travailleurs sociaux en raison du rapport de confiance qui semble s'instaurer avec les représentants religieux $^{22}$.

Face aux extérieurs et/ou aux policiers, l'établissement d'une distinction entre métiers policier et religieux en rétention repose cependant avant tout sur la mise en avant d'une frontière présentée par les aumôniers comme «morale» et/ou «éthique». Les aumôniers se considèrent ainsi, tout comme les aides-soignantes dans l'univers professionnalisé de l'hôpital étudié par Anselm L. Strauss (1992), comme «les agents principaux de l'amélioration» des conditions de vie et de l'état psychologique des étrangers, ainsi que des droits de l'homme en rétention. S'appuyant sur la « vocation à l'universel » (Agrikoliansky, 2001) que semble leur conférer leur mandat religieux supra-confessionnel, ainsi que sur la légitimité particulière dont bénéficient, dans le contexte allemand, les églises catholique et protestante en matière de défense des libertés, ils se placent

\footnotetext{
22 C'est par exemple aux aumôniers que des retenues vietnamiennes finissent par confier leur impérieux besoin de nouveaux sous-vêtements ou qu'un retenu turc fait part de la disparition, dans le colis que sa petite amie lui a adressé en rétention, des sous-vêtements féminins que cette dernière avait joints à l'envoi.
} 
de facto dans la position d' «entrepreneurs de morale» (Becker, 1985), incarnant une forme d'«humanité » (Fassin, 2010) qui transcenderait l'appareil d'État ${ }^{23}$.

«Et donc, pour moi, la première mission des aumôniers en rétention, c'est de regarder si tout est en règle ici, de faire un peu la supervision. On regarde si les gens vont à peu près bien, s'il faut intervenir sur le plan juridique - et puis, sinon, on essaye tout simplement de faire entrer un peu d'humanité dans la prison. L'Église a tout bonnement la mission de faire respecter les droits de l'homme en rétention. [...] L'Allemagne devrait être plus accueillante vis-à-vis des migrants. Ce n'est pas humain que quelqu'un qui vient d'arriver en Allemagne commence par faire l'expérience de la prison » (Markus, aumônier catholique, entretien du 30/08/2012).

Les savoirs développés au fil des ans par les aumôniers dans le domaine du conseil juridique aux étrangers reposent ainsi à la fois sur la connaissance du droit (mobilisation des savoirs «établis » des professionnels) et sur l'expérience accumulée au contact des étrangers retenus (savoirs dits «profanes ») (Lochard et Simonet, 2010). La reconnaissance qu'elle leur assure s'étend par ailleurs au-delà du groupe des retenus : outre les liens privilégiés qu'ils entretiennent avec les avocats et les associations défendant les droits des étrangers, les aumôniers sont également décrits par les autorités migratoires régionales comme des partenaires «de confiance», «très impliqués dans le processus décisionnel » institutionnel en matière d'immigration ${ }^{24}$. Exemple emblématique, la commission régionale statuant sur les «cas d'injustice flagrante» — seule instance habilitée à contester, auprès du ministre régional de l'Intérieur, le placement en rétention et/ou l'expulsion d'un étranger en situation irrégulière validés par le Tribunal administratif — compte un tiers de membres issus du clergé (catholique et protestant) : le représentant de l'Église catholique au sein de la commission, ancien aumônier au centre de Großstadt, est ainsi à lui seul à l'origine de $40 \%$ des demandes de révision déposées auprès de la commission, dont les trois-quarts environ sont ensuite validées par le ministre ${ }^{25}$.

L'acquisition de ces compétences juridiques et leur reconnaissance hors les murs permet ainsi aux aumôniers, face aux policiers, d'éviter «le rapport caritatif » et de professionnaliser leur rôle (D'Halluin-Mabillot, 2012). Le discours juridique formaliste des aumôniers, mettant en avant la nécessité de défendre les droits de retenus, est par ailleurs également un moyen de résoudre la contradiction résultant, dans l'exercice de leur mission, de leur devoir de loyauté envers les retenus et de leur activité au sein de l'institution même qui les contraint (Bouagga, 2013).

Cette contradiction apparaît d'autant plus aiguë que le processus de «professionnalisation » des aumôniers en rétention s'est accompagné ces dernières années d'une reconnaissance progressive de leur expertise non seulement hors les murs, mais aussi entre les murs de Großstadt. Cette légitimité accrue des acteurs religieux semble avoir notamment profité de l'arrivée de nouveaux acteurs au sein du dispositif policier d'aide sociale. Bien qu'ils n'aient pas tous les attributs de la profession policière (notamment le port d'armes) et ne portent désormais plus l'uniforme, les membres du service de travail social sont membres de l'institution policière, tout comme les

\footnotetext{
${ }^{23}$ L' «humanité » dont se réclament ici les acteurs religieux recouvre de fait une double dimension, «d'une part comme ensemble des êtres humains partageant une même condition (appartenir à l'humanité), et d'autre part comme mouvement affectif portant vers ses semblables (faire preuve d'humanité) » (Fassin, 2010, pp. 8-9).

${ }^{24}$ C'est notamment ce qui est ressorti de l'entretien que j'ai eu le 12/11/2012 avec un haut fonctionnaire du ministère régional de l'Intérieur en charge de la politique migratoire.

25 Source: entretien avec Josef, ancien aumônier catholique, membre de la «commission pour les cas d'injustice flagrante » en tant que représentant de l'Église catholique, 01/03/2012.
} 
médecins et infirmiers du service médical. La définition des groupes et des rôles professionnels qui prévaut intra-muros les place donc du côté de « la police» — et, partant, de la définition policière des enjeux liés à la rétention - , par opposition au groupe des aumôniers. Leur parcours professionnel s'inscrit en outre souvent dans le seul contexte d'institutions coercitives : tant le directeur du service médical qu'un travailleur social sont issus de l'institution militaire (où ils exerçaient respectivement en tant que médecin et moniteur de conduite), tandis qu'un second travailleur social n'a jamais exercé qu'en rétention. La psychologue est la seule d'entre eux à avoir forgé sa pratique professionnelle en dehors d'institutions coercitives, au sein d'un cabinet privé spécialisé dans l'accompagnement psychologique d'enfants, d'adolescents, puis de familles en difficulté. Elle a dans ce cadre accepté de dispenser certains modules de formation sur l'accompagnement psychologique des enfants et des familles au sein de l'école de police. «Motivée » par cette activité de «sensibilisation» des policiers aux spécificités du travail avec des personnes présentant des troubles psychologiques, elle a ensuite abandonné sa position au sein d'un cabinet privé pour postuler au poste de psychologue à Großstadt. Outre que son profil correspondait aux compétences exigées, la psychologue se disait motivée par le «défi » que représentait à ses yeux le fait de travailler avec des migrants. Elle est aujourd'hui directrice du service «travail social» en rétention et coordinatrice de la «commission de prévention des conflits» au sein de la police régionale. La psychologue dispose par ailleurs elle-même d'une socialisation religieuse prégnante ${ }^{26}$ dont on ne saurait exclure qu'elle ait joué un rôle dans la proximité professionnelle qu'elle revendique avec les aumôniers :

«Il s'agit de faire entendre la voix des retenus, de s'engager pour eux, de les représenter par la parole et par l'accompagnement dans ces situations de crise [...], d'exposer au retenu ses droits pour l'aider à en prendre conscience. [...] Disons que moi j'essaye de résoudre les crises par différentes techniques de distanciation et de stabilisation, et les aumôniers disposent de leurs propres instruments pour résoudre les crises, et c'est un peu notre point d'intersection, en quelque sorte, parce que finalement, les modes de résolution des crises que nous proposons ne sont pas si éloignés l'un de l'autre » (Psychologue, directrice du département policier de travail social, entretien du 23/02/2012).

Les «relations interpersonnelles inédites» (Raison du Cleuziou, 2011) ainsi permises, au sein du système de professions, entre policiers et aumôniers remettent en cause les frontières — notamment morales, mais aussi juridiques — qui séparaient jusqu'alors les groupes professionnels et la représentation de leur rôle : alors que la défense des droits des retenus et la contestation juridique du dispositif d'enfermement pouvaient jusqu'alors être considérées comme les marqueurs du positionnement des aumôniers face à l'ordre légal allemand incarné par le dispositif policier, les travailleurs sociaux de l'institution policière mettent désormais en avant la nécessité de défendre les droits des retenus et le crédit qu'ils accordent à cet égard aux aumôniers. L'observation du quotidien de la rétention témoigne ainsi de nombreux échanges d'informations entre travailleurs sociaux et/ou psychologue d'une part et aumôniers d'autre part. Ces conversations informelles mettent notamment à disposition des acteurs du travail social policier des informations sur les évolutions juridiques du statut de certains retenus, leur permettant d'adapter l'accompagnement socio-psychologique qu'ils mettent en place, et à disposition des religieux

\footnotetext{
${ }^{26}$ Elle voit ainsi dans le fait que le Saint associé à son nom soit le patron des enfants en souffrance et des prisonniers un signe du caractère quasi inéluctable de son parcours professionnel.
} 
des renseignements de nature policière (date prévue pour l'expulsion par exemple) facilitant l'ajustement de leur stratégie de défense juridique des étrangers retenus.

«Moi je fais volontiers appel aux aumôniers pour tous les problèmes d'ordre juridique [...]. Je crois que je vais d'ailleurs parfois un peu loin. Si Monsieur H. [directeur du centre de rétention] savait de quoi je parle avec les aumôniers, il ne m'adresserait plus la parole... [...] Bon, quand je flirte d'un peu trop près avec les aumôniers, Monsieur H. essaye d'une manière ou d'une autre de me freiner. [...] Il pense toujours que ces types le prennent pour un con, pour dire les choses clairement, ou qu'ils le mènent par le bout du nez. Et il ne supporte pas ça. [...] Donc quand je veux parler de quelque chose que les autres ne doivent pas entendre avec Peter B. ou Markus C., on le fait sur le palier. Ça a un côté parfois un peu conspirateur...» (Travailleur social policier, entretien du 26/01/2012).

Si la proximité revendiquée entre travailleurs sociaux de l'institution policière et aumôniers, quoique nouvelle dans le contexte rétentionnaire, peut paraître relativement peu surprenante au regard des missions d'assistance («sociale» notamment) que ces deux groupes partagent, il est plus étonnant de constater que certaines formes de reconnaissance de l'expertise religieuse se retrouvent également chez les policiers en charge de la surveillance des retenus :

«Quand on a des problèmes avec un retenu, j'en parle au pasteur, je lui dis "Pasteur, allez donc voir untel, il a des problèmes, peut-être que vous arriverez à établir le contact" » (Policier en charge de la surveillance des retenus, entretien du 16/01/2012).

Au-delà des compétences humaines qu'on leur reconnaît ici dans le rapport aux retenus, différentes déclarations des policiers laissent par ailleurs entrevoir l'attribution aux acteurs religieux d'une forme d'autorité morale sur le dispositif policier d'enfermement :

«Moi, ce que je trouve important, c'est que certains collègues soient évalués positivement par l'Église, que tout le travail ici soit reconnu par l'Église comme quelque chose de pas seulement négatif » (Policier en charge de la surveillance des retenus, entretien du 16/01/2012).

Cette autorité morale, et le rôle de « garde-fous » que joueraient par conséquent les intervenants catholiques et protestants dans les lieux d'exercice d'une contrainte étatique, sont également mis en avant par le chef du service médical policier. Bien qu'il entretienne avec les religieux un conflit permanent quant aux formes et aux principes d'exercice de la médecine en rétention, cet ancien médecin militaire compare leur autorité à celle détenue par le plus haut niveau hiérarchique dans la pratique médicale:

«Ce qui est un peu difficile avec les aumôniers, c'est qu'on a parfois le sentiment que, euh... ils ne remplissent pas leurs fonctions mais celles des autres. [...] La seule chose à laquelle je réagisse vraiment de manière allergique, c'est quand ils essayent de jouer les psychologues, les psychiatres ou les médecins. [...] Ils sont parfois pénibles, mais ça ne veut pas dire qu'ils ne soient pas quand même nécessaires. Si j'ai le choix, je préfère que les aumôniers soient là, quitte à être en conflit avec eux, plutôt que de ne pas les avoir et de risquer, un jour ou l'autre, de développer certaines formes d'aveuglement dans le travail. C'est toujours bon d'avoir quelqu'un qui observe avec méfiance ce que vous faites, parce que vous ne pouvez alors pas vous permettre d'être négligent. Je dirais que c'est comme un bon médecin-chef : il vous laisse faire, mais il surveille régulièrement et il vous fait sentir qu'il surveille » (Directeur du service médical policier, entretien du 31/10/2012). 


\section{Conclusion}

L'observation des rapports entre policiers et religieux dans l'univers professionnel de la rétention en Allemagne donne à voir la manière dont les aumôniers catholiques et protestants ont progressivement gagné en autonomie au sein d'une institution consacrant pourtant le primat policier sur l'ordre des lieux. La négociation de marges de manœuvre par les aumôniers dans l'exercice de leur mandat religieux semble avoir profité d'une « expertification » de leur rôle, notamment par le biais de leur engagement juridique en faveur des droits des retenus, ainsi que d'une humanitarisation et d'une politisation de leur position, pour les retenus et «contre le système » policier. La tension entre sécuritaire et humanitaire, entre coercition et soin, ou entre ordre et bienveillance reste donc le principe organisateur du dispositif d'enfermement de Großstadt, comme de l'ensemble des institutions étatiques de «gouvernement de la précarité » (Fassin et al., 2013). Cependant, si la définition concurrentielle des rôles et la poursuite d'objectifs a priori inconciliables entre policiers et religieux constituent la matrice dominante, garante de la légitimité professionnelle de chacun des groupes, l'ethnographie de leurs interactions révèle des rapports ambivalents entre agents de l'État et «gardiens des droits de l'homme»: l'investissement, par les acteurs policiers, du registre «humanitaire» pour justifier leur pratique professionnelle en rétention et les formes de reconnaissance policière de l'expertise et de l'autorité religieuse sur le dispositif policier, semblent ainsi permettre de poser l'hypothèse d'un rapprochement des répertoires religieux et policiers dans le contexte rétentionnaire de Großstadt. Au-delà des murs, le crédit accordé à la figure de l'expert religieux par les instances du contrôle migratoire régional atteste de leur statut d'acteurs «interdépendants — à la fois en concurrence et en coopération - des professionnels et des pouvoirs publics » (Lochard et Simonet, 2010). L'intervention d'acteurs religieux dans les murs du lieu d'enfermement, et leur constitution en experts (juridiques notamment), semblent ainsi avoir eu pour effet l'émergence, entre acteurs de l'enfermement et intervenants religieux, de «communautés morales paradoxales » (Fischer et Makaremi, 2012). Celles-ci questionnent alors les incidences de l'engagement professionnel au sein d'une institution coercitive non seulement sur les pratiques des aumôniers dans l'aide aux retenus ${ }^{27}$ — contre ou «tout contre » l'État ? (Fassin, 2006) - , mais aussi sur leur système de profession : ici, en dépit même de la transcendance divine invoquée par les acteurs religieux de Großstadt pour asseoir leur autorité morale sur les lieux, ces derniers font finalement du droit un principe supérieur à l'autorité suprême, celle de Dieu, censée gouverner leurs conduites.

\section{Déclaration d'intérêts}

L'auteur déclare ne pas avoir de conflit d'intérêts en relation avec cet article.

\section{Remerciements}

Outre les évaluateurs anonymes de la revue, je tiens à remercier Jérémie Gauthier, Fabien Jobard, Ariane Jossin et Sarah Mazouz pour leurs commentaires avisés, ainsi que les membres du

\footnotetext{
27 L'observation des pratiques d'accompagnement juridique des retenus par les aumôniers de Großstadt témoigne ainsi de tentatives de mise en conformité des migrants enfermés avec certaines assignations (genrées ou raciales notamment) produites par les instances étatiques de contrôle migratoire et relatives, en particulier, à l'incorporation des étrangers dans la nation (voir Darley, 2014).
} 
programme TerrFerme (ANR/Conseil Régional d'Aquitaine) dans le cadre duquel les enquêtes ont été réalisées.

\section{Références}

Abbott, A., 1988. The System of Professions: An Essay on the Division of Expert Labor. University of Chicago Press, Chicago.

Agrikoliansky, E., 2001. Carrières militantes et vocation à la morale : les militants de la LDH dans les années 1980. Revue française de science politique 51 (1), 27-46.

Becci, I., 2008. Collapse and Creation: The Rise and Fall of Religion in East German Offender Rehabilitation Programmes. Max Planck Institute for Social Anthropology, Halle/Saale.

Becci, I., Willems, J., 2009. Gefängnisseelsorge in der sich wandelnden ostdeutschen Gesellschaft. Eine Analyse der kulturelle, theologischen und sozialen Spannungen. International Journal of Practical Theology 13 (1), 90-120.

Becker, H.S., 1985. Outsiders. Études de sociologie de la déviance. Métailié, Paris.

Beckford, J.A., 2001. Doing Time: Space, Time, Religious Diversity and the Sacred in Prisons. International Review of Sociology 11 (3), 371-382.

Benguigui, G., Chauvenet, A., Orlic, F., 1994. Les surveillants de prison et la règle. Déviance et société 18 (3), $275-294$.

Bittner, E., 2001. Florence Nightingale à la poursuite de Willie Sutton. Regard théorique sur la police. Déviance et Société 25 (3), 285-305.

Bouagga, Y., Thèse de sociologie 2013. Humaniser la peine ? Ethnographie du traitement pénal en maison d'arrêt. EHESS, Paris.

Bourdieu, P., 1971. Genèse et structure du champ religieux. Revue française de sociologie 12 (3), 295 -334.

Chappe, V.-A., 2010. La qualification juridique est-elle soluble dans le militantisme? Tensions et paradoxes au sein de la permanence juridique d'une association antiraciste. Droit et société 3 (76), 543-567.

Chauvenet, A., 1993. Les surveillants de prison : le prix de la sécurité. Revue française de sociologie 34 (3), $345-366$.

Darley, M., Thèse de science politique 2008. Asile, frontière et détention des étrangers. Le contrôle étatique de l'immigration et son contournement en Autriche et en République tchèque. IEP, Paris.

Darley, M., 2012. «Trouvez-vous une femme ici et tout s'arrangera. . . » L'intervention religieuse auprès d'étrangers placés en rétention. Genre, sexualité \& société 8. En ligne : http://gss.revues.org/2523; DOI: 10.4000/gss.2523.

Darley, M., 2014. Les coulisses de la nation. Assignations genrées et racialisées dans les pratiques d'assistance aux étrangers en situation irrégulière. Sociétés contemporaines 94, 19-39.

D’Halluin-Mabillot, E., 2012. Les épreuves de l'asile. Associations et réfugiés face aux politiques du soupçon. Éditions de l'EHESS, Paris.

Dubois, V., 2003. La vie au guichet. Relation administrative et traitement de la misère. Economica, Paris.

Fassin, D., 2006. L'humanitaire contre l'État, tout contre. Vacarme 34, 15-19.

Fassin, D., 2010. La raison humanitaire. Une histoire morale du temps présent. Éditions de l'EHESS, Paris.

Fassin, D., Bouagga, Y., Coutant, I., Eideliman, J.-S., Fernandez, F., Fischer, N., Kobelinsky, C., Makaremi, C., Mazouz, S., Roux, S., 2013. Juger, Réprimer, Accompagner. Essai sur la morale de l'État. Seuil, Paris.

Fischer, N., 2005. Clandestins au secret. Contrôle et circulation de l'information dans les centres de rétention administrative français. Cultures \& conflits 57, 91-118.

Fischer, N., 2009. Jeux de regards. Surveillance disciplinaire et contrôle associatif dans les centres de rétention administrative. Genèses 75, 45-65.

Fischer, N., Makaremi, C., 2012. «L'horreur de la République ». Les enjeux moraux de l'enfermement des étrangers. In: Fassin, D., Eideliman, J.-S. (Eds.), Économies morales contemporaines. La Découverte, Paris, pp. $217-241$.

Goffman, E., 1968. Asiles. Études sur la condition sociale des malades mentaux. Éditions de Minuit, Paris.

Hamidi, C., 2006. Eléments pour une approche interactionniste de la politisation. Engagement associatif et rapport au politique dans des associations locales issues de l'immigration. Revue française de science politique 56 (1), 5-25.

Hughes, E.C., 1996. Le regard sociologique. Essais choisis. Éditions de l'EHESS, Paris.

Israël, L., 2003. Faire émerger le droit des étrangers en le contestant, ou l'histoire paradoxale des premières années du GISTI. Politix 62, 115-143.

Lagroye, J., 2003. Les processus de politisation. In: Lagroye, J. (Ed.), La politisation. Belin, Paris, pp. 359-372.

Lagroye, J., 2006. La vérité dans l'Église catholique. Contestations et restauration d'un régime d'autorité. Belin, Paris.

Lochard, Y., Simonet, M., 2010. Les experts associatifs, entre savoirs profanes, militants et professionnels. In: Demazière, D., Gadéa, C. (Eds.), Sociologie des groupes professionnels. La Découverte, Paris, pp. 274-284. 
Malochet, G., 2004. À l'école de la détention: quelques aspects de la socialisation professionnelle des surveillants de prison. Sociologie du travail 46 (2), 168-186.

Molinier, P., Laugier, S., Paperman, P., 2009. Qu'est-ce que le care ? Souci des autres, sensibilité, responsabilité. Éditions Payot \& Rivages, Paris.

Monjardet, D., 1996. Ce que fait la police. Sociologie de la force publique. La Découverte, Paris.

Raison du Cleuziou, Y., 2011. Le Secours Catholique et les forums sociaux : une politisation incrémentale (2003-2010). Critique internationale 50, 73-89.

Sarg, R., Lamine, A.-S., 2011. La religion en prison. Norme structurante, rehabilitation de soi, stratégie de résistance. Archives de sciences sociales des religions 153, 85-104.

Sawicki, F., 2003. Les temps de l'engagement. À propos de l'institutionnalisation d'une association de défense de l'environnement. In: Lagroye, J. (Ed.), La politisation. Belin, Paris, pp. 123-146.

Siméant, J., 1993. La violence d'un répertoire. Les sans-papiers en grève de la faim. Cultures \& conflits 9-10, 315-338.

Siméant, J., 1998. La cause des sans-papiers. Presses de Sciences Po, Paris.

Strauss, A., 1992. L'hôpital et son ordre négocié. In: Strauss, A. (Ed.), La trame de la négociation. Sociologie qualitative et interactionnisme (textes réunis et présentés par Isabelle Baszanger). L'Harmattan, Paris, pp. 87-112.

Thériault, B., 2006. Lundi à Leipzig. Les acteurs religieux en Allemagne de l'Est et la nostalgie du politique après 1989. Sociologie et sociétés 38 (1), 165-181.

Vermeersch, S., 2004. Entre individualisation et participation: l'engagement associatif bénévole. Revue française de sociologie 45 (4), 681-710.

Voßkamp, S., 2007. Katholische Kirche und Vertriebene in Westdeutschland. Integration, Identität und ostpolitischer Diskurs 1945 bis 1972. Kohlhammer, Stuttgart.

Weller, J.-M., 1994. Le mensonge d'Ernest Cigare. Problèmes épistémologiques et méthodologiques à propos de l'identité. Sociologie du travail 46 (1), 25-42. 